\title{
Modelling and analysis of a within-host model of hepatitis B and D co-infections
}

\author{
Plaire Tchinda Mouofo*, Jean Jules Tewa ${ }^{\dagger}$, Samuel Bowong ${ }^{\ddagger}$ \\ * Department of Mathematics, University of Yaounde I, \\ P.O. Box 812 Yaounde, Cameroon \\ tchindaplaire@yahoo.fr \\ $\dagger$ Department of Mathematics and Physics, \\ National Advanced School of Engineering (Polytechnic), \\ University of Yaounde I, P.O. Box 8390 Yaounde, Cameroon \\ tewajules@gmail.com \\ $\ddagger$ Department of Mathematics and Computer Science, Faculty of Science, \\ University of Douala, PO Box 24157 Douala, Cameroon \\ sbowong@gmail.com
}

Received: 17 July 2017, accepted: 21 July 2018, published: 7 August 2018

\begin{abstract}
The Hepatitis delta virus (HDV) is a defect RNA virus that requires the presence of the hepatitis B virus (HBV) for cellular infection. A coinfection may result in a more severe acute disease and a higher risk of developing acute liver failure compared with those infected with $\mathrm{HBV}$ alone. At the present time, there has been very little to the modeling of HDV. The derivation and analysis of such a mathematical model poses difficulty as it requires the inclusion of $(\mathrm{HBV})$. In this paper, a within-host model for the co-interaction of HDV and HBV is presented and rigorously analyzed. We calculate the basic reproduction number $\left(\mathcal{R}_{0}\right)$, the disease-free equilibrium, boundary equilibrium, which we define as the existence of one disease along with the complete eradication of the other disease, and the co-infection equilibrium. We determine stability criteria for the disease-free and boundary
\end{abstract}

equilibrium. We also use the optimal control theory to assess the disease control. Numerical simulations have been presented to illustrate analytical results.

Keywords-Hepatitis D, Hepatitis B, Immune system, Basic reproduction number, Optimal control.

AMS subject classifications: 34A34, 34D23, 34D40, 92D30

\section{INTRODUCTION}

Hepatitis D is a liver disease caused by the hepatitis D virus (HDV), a defective virus that needs the hepatitis B virus to exist. The hepatitis $\mathrm{D}$ virus requires the outer coating of the hepatitis B virus called the surface antigen in order to reproduce itself in a human host. The virus currently

Copyright: (C) 2018 Mouofo et al. This article is distributed under the terms of the Creative Commons Attribution License (CC BY 4.0), which permits unrestricted use, distribution, and reproduction in any medium, provided the original author and source are credited.

Citation: Plaire Tchinda Mouofo, Jean Jules Tewa, Samuel Bowong, Modelling and analysis of a within-host model of hepatitis B and D co-infections, Biomath 7 (2018), 1807219, 
infects 15 million worldwide, nearly all adults, and it is most common among injecting drug user populations and in countries bordering the Mediterranean Sea. HDV is transmitted through blood and body fluids, similar to the hepatitis B virus.

There are two types of HDV infection, coinfection and super-infection. Co-infection occurs when a patient is simultaneously infected with HDV and HBV. The majority of these patients completely recover but there is a higher rate of fulminant hepatic failure and death than with HBV infection alone. Super-infection occurs when someone with an existing chronic HBV infection becomes infected with HDV. These patients usually experience a sudden worsening of liver disease. Patients with hepatitis B who become chronically infected with HDV experience a very high rate of cirrhosis and end stage liver disease, which makes this super-infection a very dangerous disease.

There is no specific treatment for HDV infection. The most common therapeutic approach is based on the administration of interferon- $\alpha$. However, the clinical response is variable, and in most cases reversible upon interruption of treatment [1], [2]. The concomitant use of antiviral drugs like ribavirin or lamivudine, showed no significant benefits in the treatment of hepatitis delta patients [3], [4]. Although these drugs may have some inhibitory effect on HBV replication, they do not suppress HDV replication probably due to the fact that HBsAgs expression, at least in part, seems not to be affected.

The use of mathematical models to study dynamics of virus infections may represent a powerful approach to simulate the course of infection and predict the potential response to different therapies. They have been previously developed for a number of pathologies including HBV and $\mathrm{HCV}$ [5], [6], [7], [8], [9], [11], [12], [34]. the humoral immune response is universal and necessary to eliminate or control the disease after viral infection [23]. Therefore, several mathematical models have been proposed to describe the virus dynamics with humoral immunity [24], [25], [26], [27], [28], [30], [31], [32]. Mostafa et al. introduce an improved HBV model with standard incidence function, cytotoxic $\mathrm{T}$ lymphocytes (CTL) immune response, and take into account the effect of the export of precursor CTL cells from the thymus and the role of cytolytic and noncytolytic mechanisms [33]. Hattaf et al. [29] study the global stability of a generalized model of a viral dynamic that includes the adaptive immune response, represented by Cytotoxic Lymphocyte T-cell (CTL-cell ). So, their work does not take in consideration the role of the innate immune response. Noura Yousfi et al. [38] investigate a new mathematical model that describes the interactions between Hepatitis B virus (HBV), liver cells (hepatocytes), and the adaptive immune response.

More recently, the numerical simulation of the spread of HDV and HBV in a population was reported [13]. However, a mathematical model to study HDV and HBV dynamics in infected individuals is still lacking. Moreover, to our knowledge, none of the existing models take into account the reaction of immune CTL cells. The main interest in studying HBV and HDV model is to understand the long and short term behavior of the dynamics of both diseases and to predict whether the diseases will die out or will persist.

In this paper, the dynamical behavior of a cointeraction of HDV and HBV virus model with CTL immune responses is studied. The main objective is in carrying out a detailed qualitative analysis of the resulting model. The existence and the uniqueness results of the solution are discussed. We compute the basic reproduction number. We also investigate the existence of equilibria and study their stability. The model is used to determine the optimal methodology for administering anti-viral medication therapies to fight HBV and HDV infection. In particular, we investigates the fundamental role of chemotherapy treatment in controlling the virus reproduction. A characterization of the optimal control via adjoint variables is also established. We obtain an opti- 
mality system that we seek to solve numerically. It is our view that this study represents the very first modelling work that provides an in-depth analysis of the qualitative dynamics of HBV-HDV co-infection and its control.

The paper is organized as follows. In the next section, we propose a new mathematical model for $\mathrm{HBV}$ infection alone that takes into account the CTL immune system. To be more realistic, we have assumed that the infection rate is given by the standard incidence function [16]. The model is rigorously analyzed. In Section 3, we formulate and analyze a realistic mathematical model for HBV-HDV co-infection, which incorporates the key epidemiological and biological features of each of the two diseases. Optimal control approach is applied in Section 4, in order to find the best way to fight the co-infection between the two diseases. We end this paper with a brief discussion and conclusion.

\section{THE HBV MODEL}

\section{A. The model description}

We consider the HBV model given by the following differential equations:

$$
\left\{\begin{array}{l}
\dot{x}=\lambda-d_{x} x-\frac{\beta(1-\eta) x v}{x+y} \\
\dot{y}=\frac{\beta(1-\eta) x v}{x+y}-a_{y} y-\delta y I \\
\dot{v}=k(1-\varepsilon) y-d_{v} v \\
\dot{I}=\rho y I+p I-q I^{2}
\end{array}\right.
$$

where $x$ is the number of uninfected liver cells, $y$ is the number of infected liver cells, $v$ is the number of free virus, and $I$ is the number of CTL cells. All the parameters $\lambda, d_{x}, \eta, \beta, a_{y}$, $\delta, \varepsilon, k, d_{v}, \rho, p$, and $q$ are positive. $d_{x}, a_{y}$ and $d_{v}$ are the death rates of uninfected liver cells, the infected cells and free virus, respectively. The constant parameter $\lambda$ represents the production of the liver cells. $\beta$ is the contact rate between uninfected cells and free virus. Free virus is produced from infected cells at rate $k y$. Infected cells are removed at rate $\delta I$ by CTL immune responses. The virus-specific CTL cells proliferate at rate $\rho y$ by contacts with infected cells. The parameter $\eta$ is the efficacy of inhibiting new virus infections as a consequence of virus clearance and $\varepsilon$ the efficacy of inhibiting viral production from infected cells. Of course the behaviour when there is no treatment is obtained by setting $\eta=\varepsilon=0$. The parameter $p$ denotes the proliferation rate of immune cells and $q$ the density-dependent rate of immune cells suppression. more precisely, we suppose that the immune cells expand at a net rate $p$, which encapsulate the positive feedback upon the immune system. The parameter $q$ comes from the fact that we assume a regulatory negative feedback force such as the effect of cell density, inhibitory cytokines or natural apoptosis, which oparates to suppress immune population growth. we suppose in this case that immune population is suppressed at a net rate $q$ which is proportional to the square of its density $\left(q I^{2}\right)$. So, the term $p I-q I^{2}$ can be written as $p I\left(1-\frac{I}{\frac{p}{q}}\right)$ which express a logistic law for evolution of immune population in the absence of infected cells.

\section{B. Analysis of the model}

Herein, we present some basic results, such as the positive invariance of model system (1), the boundedness of solutions, the existence of equilibria and and its stability analysis.

\section{1) Positivity and boundedness of solutions:}

The following result guarantees that model system (1) is biologically well behaved and its dynamics is concentrated on a bounded region of $\mathbb{R}_{+}^{4}$. More precisely, the following result holds.

Theorem 1. Let $R_{+}^{4}=\left\{(x, y, v, I) \in \mathbb{R}^{4}: x \geq\right.$ $0, y \geq 0, v \geq 0, I \geq 0\}$. Then, $\mathbb{R}_{+}^{4}$ is positively invariant under the flow induced by model system (1). Moreover, the region

$$
\begin{aligned}
& \Delta=\left\{(x, y, v, I) \in \mathbb{R}^{4}: x+y \leq \frac{\lambda}{d_{x}},\right. \\
&\left.v \leq \frac{k \lambda(1-\varepsilon)}{d_{x} d_{v}}, \frac{p}{q} \leq I \leq \frac{p+\frac{\rho \lambda}{d_{x}}}{q}\right\}
\end{aligned}
$$

is positively invariant and absorbing with respect to model system (1). 
P. T. Mouofo, J. J. Tewa, S. Bowong, Modelling and analysis of a within-host model of hepatitis ...

Proof: No solution of model system (1) with initial conditions $(x(0), y(0), v(0), I(0)) \in \mathbb{R}_{+}^{4}$ is negative. In fact, for $(x(t), y(t), v(t), I(t)) \in \mathbb{R}_{+}^{4}$, we have $\left.\dot{x}\right|_{x=0}=\lambda>0,\left.\dot{y}\right|_{y=0}=(1-$ 7) $\beta v \geq 0,\left.\dot{v}\right|_{v=0}=(1-\varepsilon) k \geq 0,\left.\dot{I}\right|_{I=0}=$ $0 \geq 0$, this immediately implies that all solutions of model system (1) with initial condition $(x(0), y(0), v(0), I(0)) \in \mathbb{R}_{+}^{4}$ stay in the first quadrant.

For the invariance property of $\Delta$, it suffices to show that the vector field, on the boundary, does not point to the exterior. Adding the first and second equations of model system(1) yields on the boundary of $\Delta$ :

$$
\begin{aligned}
& \left.\frac{d(x+y)}{d t}\right|_{x+y=\frac{\lambda}{d_{x}}} \\
& =\lambda-d_{x} x-a_{y} y-\left.\delta y I\right|_{x+y=\frac{\lambda}{d_{x}}} \\
& \leq\left.\left(\lambda-d_{x}(x+y)\right)\right|_{x+y=\frac{\lambda}{d_{x}}}=0 .
\end{aligned}
$$

Similarly, we get

$$
\begin{aligned}
& \left.\frac{d v}{d t}\right|_{v=\frac{k \lambda(1-\varepsilon)}{d_{x} d_{v}}} \quad \frac{k \lambda(1-\varepsilon)}{d_{x}}-\left.d_{v} v\right|_{v=\frac{k \lambda(1-\varepsilon)}{d_{x} d_{v}}}=0 \\
& \left.\frac{d I}{d t}\right|_{I=\frac{p}{q}} \geq\left.(p-q I) I\right|_{I=\frac{p}{q}}=0, \\
& \quad \text { i.e } I(t) \geq \frac{p}{q} \forall t \in[0,+\infty)
\end{aligned}
$$

and

$$
\begin{aligned}
& \left.\frac{d I}{d t}\right|_{I=\frac{p+\frac{\rho \lambda}{\mu}}{q}} \\
& \quad \leq\left.\left(\frac{\rho \lambda}{d_{x}}+p-q I\right) I\right|_{I=\frac{p+\frac{\rho \lambda}{\mu}}{q}}=0 .
\end{aligned}
$$

Therefore, solutions starting in $\Delta$ will remain there for $t \geq 0$.

Now, we prove the attractiveness of the trajectories of model system (1). To do so, from model system (1), one has

$$
\frac{d(x+y)}{d t} \leq \lambda-d_{x}(x+y) . \quad \mathcal{R}_{0}=\frac{k(1-\varepsilon) \beta(1-\eta)}{d_{v}\left(a_{y}+\delta I^{*}\right)}
$$

$$
F=\left(\begin{array}{cc}
0 & \beta(1-\eta) \\
k(1-\varepsilon) & 0
\end{array}\right)
$$

and

$$
V=\left(\begin{array}{cc}
a_{y}+\delta I^{*} & 0 \\
0 & d_{v}
\end{array}\right)
$$

Thus, the basic reproduction number is given by: 
P. T. Mouofo, J. J. Tewa, S. Bowong, Modelling and analysis of a within-host model of hepatitis ...

From theorem 2 of Van Den Driessche[17], we have the following result.

Lemma 1. The virus-free equilibrium $P_{1}$ of the model system (1) is locally asymptotically stable (LAS) if $\mathcal{R}_{0}<1$, and unstable if $\mathcal{R}_{0}>1$.

We now study the existence of equilibria of model system (1). Setting the right-hand sides of model system (1) equals to zero gives

$$
\begin{gathered}
\lambda-d_{x} x-\frac{\beta(1-\eta) x v}{x+y}=0, \\
\frac{\beta(1-\eta) x v}{x+y}-a_{y} y-\delta y I=0, \\
k(1-\varepsilon) y-d_{v} v=0, \\
\rho y I+p I-q I^{2}=0 .
\end{gathered}
$$

Model system (1) has always equilibrium $P_{1}=$ $\left(x^{*}, 0,0, I^{*}\right)$ which is the virus free equilibrium and represents the state when the viruses are absent. To find the endemic equilibrium, we look for $P_{2}=(\bar{x}, \bar{y}, \bar{v}, \bar{I})$ that represents the state in which both the viruses and CTL cells are present. Let $\gamma=\frac{d_{v}}{k(1-\varepsilon)}, a=\frac{\rho d_{v}}{q k(1-\varepsilon)}, b=\frac{p}{q}$ and assume that $\mathcal{R}_{0}>1$. From Eqs.(5) and (6), one obtains

$$
\bar{y}=\gamma \bar{v} \quad \text { and } \quad \bar{I}=a \bar{v}+b .
$$

Substituting the above expression of $\bar{y}$ in Eq. (4) yields

$$
\bar{v}\left(\frac{\beta(1-\eta) \bar{x}}{\bar{x}+\gamma \bar{v}}-a_{y} \gamma-\delta \gamma(a \bar{v}+b)\right)=0
$$

Since $\bar{v} \neq 0$, Eq. (8) leads to

$$
\bar{x}=\frac{\gamma^{2} \bar{v}\left(a_{y}+\delta b+\delta a \bar{v}\right)}{\beta(1-\eta)-\gamma\left(a_{y}+\delta b+\delta a \bar{v}\right)} .
$$

Since $\bar{x}>0$, one can deduce that

$$
\bar{v}<\frac{\beta(1-\eta)-\gamma\left(a_{y}+\delta b\right)}{\gamma \delta a} .
$$

Note that

$$
\beta(1-\eta)-\gamma\left(a_{y}+\delta b\right)=\frac{d_{v}\left(a_{y}+\delta b\right)}{k(1-\varepsilon)}\left[\mathcal{R}_{0}-1\right] .
$$

Thus, $\bar{x}>0$ implies that $v \in] 0, \tilde{v}_{1}[$, where

$$
\tilde{v}_{1}=\frac{d_{v}\left(a_{y}+\delta b\right)}{\gamma \delta a k(1-\varepsilon)}\left[\mathcal{R}_{0}-1\right] .
$$

Moreover, since

$$
\bar{x}<\frac{\lambda}{d_{x}},
$$

the above relation implies that

$$
\begin{array}{r}
\gamma^{2} \delta a d_{x} \bar{v}^{2}+\left[d_{x} \gamma^{2}\left(a_{y}+\delta b\right)+\lambda \gamma \delta a\right] \bar{v} \\
-\lambda\left[\beta(1-\eta)-\gamma\left(a_{y}+\delta b\right)\right]<0
\end{array}
$$

Since $\mathcal{R}_{0}>1$, the discriminant of 111 is

$$
\begin{aligned}
\text { delta }= & {\left[d_{x} \gamma^{2}\left(a_{y}+\delta b\right)+\lambda \gamma \delta a\right]^{2} } \\
& +4 \lambda \gamma^{2} \delta a d_{x}\left[\beta(1-\eta)-\gamma\left(a_{y}+\delta b\right)\right]>0 .
\end{aligned}
$$

Thus, the condition $\bar{x}<\frac{\lambda}{d_{x}}$ implies that $\left.\bar{v} \in\right] 0, \tilde{v}_{2}[$ where

$$
\tilde{v}_{2}=\frac{-\left[d_{x} \gamma^{2}\left(a_{y}+\delta b\right)+\lambda \gamma \delta a\right]+\sqrt{\text { delta }}}{2 \gamma^{2} \delta a d_{x}}
$$

We have the following result.

Lemma 2. Let $\tilde{v}_{1}$ and $\tilde{v}_{2}$ given in Eqs. (10) and (12), respectively. Then, $\tilde{v}_{2}<\tilde{v}_{1}$ whenever $\mathcal{R}_{0}>$ 1.

Proof: Since $\mathcal{R}_{0}>1$, applying some technical manipulation one can show that

$\gamma d_{x}\left[\beta(1-\eta)-\gamma\left(a_{y}+\delta b\right)\right]+d_{x} \gamma^{2}\left[a_{y}+\delta b\right]>0$

is equivalent to

$$
\tilde{v}_{1}>\tilde{v}_{2}
$$

Remark 1. 1) From Lemma 2 conditions $\bar{v} \in$ ]0, $\tilde{v}_{1}[$ and $\bar{v} \in] 0, \tilde{v}_{2}[$ can be limited to $\bar{v} \in$ ] $0, \tilde{v}_{2}\left[\right.$. Thus, when $\mathcal{R}_{0}>1$, model system (1) may have an endemic equilibrium $P_{2}=$ $(\bar{x}, \bar{y}, \bar{v}, \bar{I})$ with $\bar{v} \in] 0, \tilde{v}_{2}[$.

2) We point out that $\bar{v}=\tilde{v}_{2}$ implies that $\bar{x}=$ $\frac{\lambda}{d_{x}}$.

Substituting Eq. (7) and Eqs. (9) into (3), we obtain the following cubic equation in $\bar{v}$ :

$$
a_{3} \bar{v}^{3}+a_{2} \bar{v}^{2}+a_{1} \bar{v}+a_{0}=0,
$$


P. T. Mouofo, J. J. Tewa, S. Bowong, Modelling and analysis of a within-host model of hepatitis ...

where

$$
\begin{aligned}
a_{3}= & {\left[\frac{d^{2} \delta \rho}{q k^{2}(1-\varepsilon)^{2}}\right]^{2}, } \\
a_{2}= & \frac{d_{v}^{2}\left(a_{y}+\delta \frac{p}{q}\right)}{k^{2}(1-\varepsilon)^{2}}-\frac{d_{x} \delta \rho d_{v}^{3}}{q k^{3}(1-\varepsilon)^{3}} \\
& -\frac{\delta \rho d_{v}^{3}\left(a_{y}+\delta \frac{p}{q}\right)}{q k^{3}(1-\varepsilon)^{3}}\left[\mathcal{R}_{0}-1\right], \\
a_{1}= & -\frac{\lambda d_{v}^{2} \delta \rho}{q k^{2}(1-\varepsilon)^{2}}-\frac{d_{x} d_{v}^{2}\left(a_{y}+\delta \frac{p}{q}\right)}{k^{2}(1-\varepsilon)^{2}} \\
& -\frac{\gamma d_{v}\left(a_{y}+\delta \frac{p}{q}\right)^{2}}{k(1-\varepsilon)}\left[\mathcal{R}_{0}-1\right], \\
a_{0}= & \frac{\lambda d_{v}\left(a_{y}+\delta \frac{p}{q}\right)}{k(1-\varepsilon)}\left[\mathcal{R}_{0}-1\right] .
\end{aligned}
$$

The coefficient $a_{3}$ is always positive. Also, if $\mathcal{R}_{0}>1$, then $a_{0}>0$ and $a_{1}<0$. Using the Descartes' rules of sign, if $\mathcal{R}_{0}>1$, the above equation given in (14) has exactly nought or two positive solutions. Let us consider the following polynomial

$$
\begin{aligned}
P:\left[0, \tilde{v}_{2}\right] & \rightarrow \mathbb{R} \\
\bar{v} & \mapsto P(\bar{v})=a_{3} \bar{v}^{3}+a_{2} \bar{v}^{2}+a_{1} \bar{v}+a_{0} .
\end{aligned}
$$

We have

- $P(0)=a_{0}>0$ since $\mathcal{R}_{0}>1$

- $P\left(\tilde{v}_{2}\right)=-\frac{\beta(1-\eta) k(1-\varepsilon) \lambda \tilde{v}_{2}}{\lambda k(1-\varepsilon)+d_{x} d_{v} \tilde{v}_{2}}<0$

Since $P(0)>0$ and $P\left(\tilde{v}_{2}\right)<0$, Eq. 14 has only one or three solutions on $] 0, v_{2}[$. By the Descartes' rules of sign, Eq.(14) cannot have three solutions on $] 0, \tilde{v}_{2}[$.

We have proved the following result.

Theorem 2. If $\mathcal{R}_{0}>1$, model system (1) has exactly one endemic equilibrium $P_{2}=(\bar{x}, \bar{y}, \bar{v}, \bar{I})$ where $\bar{x}, \bar{y}$ and $\bar{I}$ are defined in Eqs. (7) and (9) with $\bar{v} \in] 0, \tilde{v}_{2}$ [ satisfying Eq. (14).

Remark 2. When $\mathcal{R}_{0}<1$, we have $\bar{x}<0$. So, there is no endemic equilibrium.

\section{3) Stability of equilibria:}

Here, we study the stability of equilibria.

We have the following result about the global stability of the virus free equilibrium.

Theorem 3. When $\mathcal{R}_{0}<1$, then the virus free equilibrium $P_{1}=\left(x^{*}, 0,0, I^{*}\right)$ is globally asymptotically stable in $\Delta$.

Proof: We consider the following LaSalleLyapunov candidate function:

$$
L(t)=b_{1} y+b_{2} v
$$

where $b_{1}$ and $b_{2}$ are positive constants to be determined later. Using the fact that $x \leq x^{*}$ and $I \geq I^{*}$, its time derivative along the trajectories of (1) satisfies

$$
\begin{aligned}
\dot{L}= & \left(\frac{b_{1} \beta(1-\eta) x}{x+y}-b_{2} d_{v}\right) v \\
& +\left[b_{2} k(1-\varepsilon)-b_{1}\left(a_{y}+\delta I\right)\right] y, \\
\leq & \left(\frac{b_{1} \beta(1-\eta) x^{*}}{x^{*}+y}-b_{2} d_{v}\right) v \\
& +\left[b_{2} k(1-\varepsilon)-b_{1}\left(a_{y}+\delta I^{*}\right)\right] y .
\end{aligned}
$$

The constants $b_{1}$ and $b_{2}$ are chosen such that the coefficient of $y$ are equal to zero, that is, $b_{1}=$ $k(1-\varepsilon)$ and $b_{2}=a_{y}+\delta I^{*}$. Then, we obtain

$$
\dot{L} \leq\left(a_{y}+\delta I^{*}\right) d_{v}\left(\mathcal{R}_{0}-1\right) v .
$$

Thus, $\dot{L}(t) \leq 0$ when $\mathcal{R}_{0}<1$. By LaSalle's invariance principle, the largest invariant set in $\left\{(x, y, v, I) \in \mathbb{R}_{+}^{4}, \dot{L}(t)=0\right\} \subset \Delta$ is reduced to the virus free equilibrium. This proves the global asymptotic stability of $P_{1}=\left(x^{*}, 0,0, I^{*}\right)$ on $\Delta$ [18] (Theorem 3.7.11, page 346). This achieves the proof.

Now, we study the stability of the endemic equilibrium. The Jacobian matrix of model system (1) at the endemic equilibrium $P_{2}=(\bar{x}, \bar{y}, \bar{v}, \bar{I})$ is

$$
J_{3}=\left(\begin{array}{cccc}
-d_{x}-U & W & -V & 0 \\
U & -W-a_{y}-\delta \bar{I} & V & -\delta \bar{y} \\
0 & k(1-\varepsilon) & -d_{v} & 0 \\
0 & \rho \bar{I} & 0 & -q \bar{I}
\end{array}\right)
$$


P. T. Mouofo, J. J. Tewa, S. Bowong, Modelling and analysis of a within-host model of hepatitis ...

where $U=\frac{(1-\eta) \beta \bar{v} \bar{y}}{(\bar{x}+\bar{y})^{2}}, V=\frac{(1-\eta) \beta \bar{x}}{\bar{x}+\bar{y}}$ and $W=\frac{(1-\eta) \beta \bar{v} \bar{x}}{(\bar{x}+\bar{y})^{2}}$. The characteristic equation of $J_{3}$ is

$$
\xi^{4}+c_{1} \xi^{3}+c_{2} \xi^{2}+c_{3} \xi+c_{4}=0
$$

where

$$
\begin{aligned}
c_{1} & =d_{x}+U+W+a_{y}+\delta \bar{I}+d_{v}+q \bar{I}, \\
c_{2} & =d_{x} W+d_{x} a_{y}+d_{x} \delta \bar{I}+d_{x} d_{v}+d_{x} q \bar{I} \\
& +U a_{y}+U \delta \bar{I}+U d_{v}+U q \bar{I}+W d_{v} \\
& +q \bar{I} W+q \bar{I} a_{y}+q \bar{I}^{2} \delta+q \bar{I} d_{v}+\delta \bar{y} \rho \bar{I}, \\
c_{3} & =U a_{y} d_{v}+U \delta \bar{I} d_{v}+d_{x} W d_{v}+d_{x} q \bar{I} W \\
& +d_{x} q \bar{I} a_{y}+d_{x} q \bar{I}^{2} \delta+d_{x} q \bar{I} d_{v}+d_{x} \delta \bar{y} \rho \bar{I} \\
& +U q \bar{I} a_{y}+U q \bar{I}^{2} \delta+U q \bar{I} d_{v}+U \delta \bar{y} \rho \bar{I} \\
& +W d_{v} q \bar{I}+\delta \bar{y} \rho \bar{I} d_{v} \\
c_{4} & =d_{x} W d_{v} q \bar{I}+d_{x} \delta \bar{y} \rho \bar{I} d_{v}+U \delta \bar{y} \rho \bar{I} d_{v} \\
& +U a_{y} d_{v} q \bar{I}+U \delta \bar{I}^{2} d_{v} q .
\end{aligned}
$$

It is clear that $c_{1}>0, c_{3}>0$ and $c_{4}>0$. After some technical computation, we obtain that $c_{1} c_{2} c_{3}-c_{3}^{2}-c_{1}^{2} c_{4}$ is positive. Hence, we have proved the following result.

Theorem 4. The endemic equilibrium $P_{2}$ of model system (1) is locally asymptotically stable if $\mathcal{R}_{0}>$ 1.

\section{THE HB V-HDV CO-INFECTION MODEL}

\section{A. Model construction}

In this section, we incorporate the HDV infection into the previous model in order to obtain a mathematical models that can describe the dynamics of HDV and HBV co-infection. We add three state variables, namely the HDV viral load, hepatocyte infected with HDV and those coinfected with both HBV and HDV. So, we use the following state variables:

- $x(t)$ the number of uninfected cells at time $t$,

- $y(t)$ the number of HBV infected cells at time $t$,

- $z(t)$ the number of HDV infected cells at time $t$,

- $w(t)$ the number of infected cells with both HBV and HDV at time $t$,

- $v_{1}(t)$ the HBV viral load at time $t$,

- $v_{2}(t)$ the HDV viral load at time $t$,

- $I(t)$ the number of CTL cells at time $t$.

We make the following hypothesis:

1) Uninfected liver cells $(x)$ can only be infected by HBV virions alone and become $y$, or by HDV virions alone and become $z$.

2) Infected cells by HBV virions can be superinfected by HDV and become $w$.

3) Infected cells by HDV virions can be superinfected by HBV and become $w$.

Putting the above formulations and assumptions together gives the following system of differential equations:

$$
\left\{\begin{array}{l}
\dot{x}=\lambda-d_{x} x-\frac{\beta_{1}(1-\eta) x v_{1}}{P}-\frac{\beta_{2}(1-\eta) x v_{2}}{P}, \\
\dot{y}=\frac{\beta_{1}(1-\eta) x v_{1}}{P}-\frac{\beta_{2}(1-\eta) y v_{2}}{P}-a_{y} y-\delta_{1} y I, \\
\dot{z}=\frac{\beta_{2}(1-\eta) x v_{2}}{P}-\frac{\beta_{1}(1-\eta) z v_{1}}{P}-a_{z} z-\delta_{2} z I, \\
\dot{w}=\frac{\beta_{2}(1-\eta) y v_{2}}{P}+\frac{\beta_{1}(1-\eta) z v_{1}}{P}-a_{w} w-\delta_{3} w I, \\
\dot{v}_{1}=k_{1}(1-\varepsilon) y+k_{3}(1-\varepsilon) w-d_{1} v_{1}, \\
\dot{v}_{2}=k_{2}(1-\varepsilon) w-d_{2} v_{2}, \\
\dot{I}=\rho_{1} y I+\rho_{2} z I+\rho_{3} w I+p I-q I^{2},
\end{array}\right.
$$

where

$$
P=x+y+z+w .
$$

Susceptible host (healthy hepatocytes) cells are produced at a rate $\lambda$, died at a rate $d_{x} . \beta_{1}$ and $\beta_{2}$ are respectively the contact rates between uninfected cells with free HBV virions, and uninfected cells with free HDV virions. $a_{y}, a_{z}$ and $a_{w}$ are respectively death rate of $\mathrm{HBV}$ only infected cells, HDV only infected cells and coinfected cells by HDV and HBV. In these equations, all the parameters are positive and we assume that the death rate of uninfected cells is not greater than the death rate of infected cells, that is, $\min \left\{d_{x}, a_{y}, a_{z}, a_{w}\right\}=d_{x}$. HBV virions $v_{1}$ are 
P. T. Mouofo, J. J. Tewa, S. Bowong, Modelling and analysis of a within-host model of hepatitis ...

TABLE I

NumericAl VALUES FOR THE PARAMETERS OF MODEL SYSTEM 19]

\begin{tabular}{|c|c|c|c|}
\hline Symbols & Definition & value & Source \\
\hline$\overline{\lambda \lambda}$ & Production rate of hepathocyte & 252666.6667 day $^{-1}$ & {$[\overline{10]}$} \\
\hline$d_{x}$ & Normal death rate of healthy liver cells & 0.0039 days $^{-1}$ & [15] \\
\hline$a_{y}$ & Infected cell death rate (HBV) & $0.0693-0.00693 \mathrm{day}^{-1}$ & [9] \\
\hline$a_{z}$ & Infected cell death rate (HDV) & 0.009 day $^{-1}$ & Assumed \\
\hline$a_{w}$ & Infected cell death rate (HBV-HDV) & $0.0091 \mathrm{day}^{-1}$ & Assumed \\
\hline$d_{1}$ & death rate of free $\mathrm{HBV}$ & $0.693 \mathrm{day}^{-1}$ & [14] \\
\hline$d_{2}$ & death rate of free HDV & 0.6 day $^{-1}$ & Assumed \\
\hline$\beta_{1}$ & Infection rate of $\mathrm{HBV}$ & $3.6 \times 10^{-5}-1.8 \times 10^{-3}$ & [14] \\
\hline$\beta_{2}$ & Infection rate of HDV & 0.002 day $^{-1}$ & Assumed \\
\hline \multirow[t]{2}{*}{$k_{1}$} & HBV production per infected & & \\
\hline & liver cells & $200-1000$ days $^{-1}$ & [8] \\
\hline \multirow[t]{2}{*}{$k_{2}$} & HDV production per infected & & \\
\hline & $\begin{array}{l}\text { liver cells } \\
\text { HBV production per co-infected }\end{array}$ & 300 days $^{-1}$ & Assumed \\
\hline$k_{3}$ & HBV\& HDV cells & 300 days $^{-1}$ & Assumed \\
\hline$\delta_{1}$ & $\begin{array}{l}\text { rate of CTL elimination } \\
\text { in infected HBV cells }\end{array}$ & 0.02 day $^{-1}$ & Assumed \\
\hline$\delta_{2}$ & $\begin{array}{l}\text { rate of CTL elimination } \\
\text { in infected HDV cells }\end{array}$ & 0.02 day $^{-1}$ & Assumed \\
\hline$\delta_{3}$ & $\begin{array}{l}\text { rate of CTL elimination } \\
\text { in infected HBV-HDV cells }\end{array}$ & $0.02 \mathrm{day}^{-1}$ & Assumed \\
\hline$p$ & the proliferation rate of immune cells & 0.5 day $^{-1}$ & Assumed \\
\hline$q$ & $\begin{array}{l}\text { density-dependent rate of immune } \\
\text { cells suppression }\end{array}$ & 0.03 day $^{-1}$ & Assumed \\
\hline$\rho_{i}$ & HBV-specific CTL stimulation rate & $0.02 \mathrm{day}^{-1}$ & Assumed \\
\hline
\end{tabular}

produced by HBV only infected cells $y$ and by coinfected cells $w$. In fact, in order for HDV to successfully complete its replication cycle, a hepatocyte must be coinfected with HBV and HDV. In these coinfected cells, the replication of $\mathrm{HBV}$ is suppressed by HDV, although not completely abolished [19], [20]. So, $v_{1}$ can be produced by $w$. HBV only infected cells $y$ produce HBV virions particles $v_{1}$ at a rate $k_{1}(1-\varepsilon) y$ and are killed by the CTL immune response at a rate $\delta_{1} y I$. For the same reason, HDV are killed by the CTL immune response at a rate $\delta_{1} y I$. Finally, CTL cells increase at a rates $\rho_{1} y I, \rho_{2} z I$ and $\rho_{3} w I$ as a result of stimulation by the viral antigen of the infected cells. $p$ and $q$ have the same meaning as in the previous model.

The parameter values used for numerical simulation are given in Table 1 .

\section{B. Mathematical analysis of the model}

\section{1) Positivity and boundedness of trajectories:}

We have the following result.

Theorem 5. The nonegative orthant $\mathbb{R}_{+}^{7}$ is positively invariant for model system (19). Moreover, system (19) is pointwise dissipative and the $a b$ - 
P. T. Mouofo, J. J. Tewa, S. Bowong, Modelling and analysis of a within-host model of hepatitis ...

sorbing set is given by

$$
\begin{aligned}
\Sigma=\{ & \left(x, y, z, w, v_{1}, v_{2}, I\right) \in \mathbb{R}_{+}^{7}: T \leq \frac{\lambda}{d_{x}}, \\
& v_{1} \leq \frac{\left(k_{1}+k_{3}\right)(1-\varepsilon) \lambda}{d_{x} d_{1}}, v_{2} \leq \frac{\lambda k_{2}(1-\varepsilon)}{d_{x} d_{2}}, \\
& \left.\frac{p}{q} \leq I \leq \frac{\rho_{0}}{q}\right\}
\end{aligned}
$$

where $T=x+y+z+w$ and $\rho_{0}=\frac{\left(\rho_{1}+\rho_{2}+\rho_{3}\right) \lambda}{d_{x}}+p$.

The proof is similar to the proof of Theorem 1.

2) Basic reproduction number and equilibria:

The disease-free equilibrium of model system (19) is given by $E_{0}=\left(x^{*}, 0,0,0,0,0, I^{*}\right)$.

Using the notations in van den Driessche and Watmough[17] for model system (19), the matrices $F$ and $V$ for the new infection terms and the remaining transfer terms are, respectively, given by

$$
F=\left(\begin{array}{ccccc}
0 & 0 & 0 & (1-\eta) \beta_{1} & 0 \\
0 & 0 & 0 & 0 & (1-\eta) \beta_{2} \\
0 & 0 & 0 & 0 & 0 \\
k_{1}(1-\varepsilon) & 0 & k_{3}(1-\varepsilon) & 0 & 0 \\
0 & 0 & k_{2}(1-\varepsilon) & 0 & 0
\end{array}\right)
$$

and

$$
V=\left(\begin{array}{ccccc}
a_{y}+\delta_{1} I^{*} & 0 & 0 & 0 & 0 \\
0 & a_{z}+\delta_{2} I^{*} & 0 & 0 & 0 \\
0 & 0 & a_{w}+\delta_{3} I^{*} & 0 & 0 \\
0 & 0 & 0 & d_{1} & 0 \\
0 & 0 & 0 & 0 & d_{2}
\end{array}\right) .
$$

It then follows that the basic reproduction number is given by

$$
\mathcal{R}_{0}=\frac{k_{1}(1-\varepsilon) \beta_{1}(1-\eta)}{d_{1}\left(a_{y}+\delta_{1} I^{*}\right)} .
$$

Thus, using Theorem 2 of van den Driessche and Watmough[17], we have the following result.

Lemma 3. : The virus free equilibrium $E_{0}$ of model system (19) is locally asymptotically stable (LAS) if $\mathcal{R}_{0}<1$, and unstable if $\mathcal{R}_{0}>1$.

Remark 3. Note that the basic reproduction number of the co-infection model of $H B V$ and $H D V$ is the same than the basic the basic reproduction of the HBV model alone. This suggests that to control the co-infection of $H B V$ and HDV within the body of a host, one only needs to control the $H B V$ infection.

We now process with the existence of steady states.

The steady states of model system (19) satisfy the following equations:

$$
\left\{\begin{array}{l}
\lambda-d_{x} \bar{x}-\frac{\beta_{1}(1-\eta) \bar{x} \bar{v}_{1}}{\bar{P}}-\frac{\beta_{2}(1-\eta) \bar{x} \bar{v}_{2}}{\bar{P}}=0, \\
\frac{\beta_{1}(1-\eta) \bar{x} \bar{v}_{1}}{\bar{P}}-\frac{\beta_{2}(1-\eta) \bar{y} \bar{v}_{2}}{\bar{P}}-a_{y} \bar{y}-\delta_{1} \bar{y} \bar{I}=0, \\
\frac{\beta_{2}(1-\eta) \bar{x} \bar{v}_{2}}{\bar{P}}-\frac{\beta_{1}(1-\eta) \bar{z} \bar{v}_{1}}{P}-a_{z} \bar{z}-\delta_{2} \bar{z} \bar{I}=0, \\
\frac{\beta_{2}(1-\eta) \bar{v}_{2}}{\bar{P}}+\frac{\beta_{1}(1-\eta) \bar{z} \bar{v}_{1}}{\bar{P}}-a_{w} \bar{w}-\delta_{3} \bar{w} \bar{I}=0, \\
k_{1}(1-\varepsilon) \bar{y}+k_{3}(1-\varepsilon) \bar{w}-d_{1} \bar{v}_{1}=0, \\
k_{2}(1-\varepsilon) \bar{w}-d_{2} \bar{v}_{2}, \\
\rho_{1} \bar{y} \bar{I}+\rho_{2} \bar{z} \bar{I}+\rho_{3} \bar{w} \bar{I}+p \bar{I}-q \bar{I}^{2}=0,
\end{array}\right.
$$

where $\bar{P}=\bar{x}+\bar{y}+\bar{z}+\bar{w}$. From the last equation of (22), we have

$$
\left(\rho_{1} \bar{y}+\rho_{2} \bar{z}+\rho_{3} \bar{w}+p-q \bar{I}\right) \bar{I}=0,
$$

which has two possible solutions: $\bar{I}=0$ and $\rho_{1} \bar{y}+$ $\rho_{2} \bar{z}+\rho_{3} \bar{w}+p-q \bar{I}=0$. Since $\bar{I} \geq I^{*}$ and $\bar{I} \neq 0$, one has that $\bar{I}=\frac{1}{q}\left(\rho_{1} \bar{y}+\rho_{2} \bar{z}+\rho_{3} \bar{w}+p\right)$. From the sixth equation of 22 , one has $\bar{w}=\frac{d_{2}}{k_{2}(1-\varepsilon)} \bar{v}_{2}$. Adding the third and fourth equations of (23) and using the expression of $\bar{w}$ yields

$$
\bar{z}=\left[\frac{\beta_{2}(1-\eta)(\bar{x}+\bar{y})}{\left(a_{z}+\delta_{2} \bar{I}\right) \bar{P}}-\frac{\left(a_{w}+\delta_{3} \bar{I}\right) d_{2}}{k_{2}(1-\varepsilon)\left(a_{z}+\delta_{2} \bar{I}\right)}\right] \bar{v}_{2} .
$$

Substituting Eq. (24) into the fourth equation of (22) gives

$$
\begin{aligned}
\left\{\frac{\beta_{2}(1-\eta) \bar{y}}{\bar{P}}+\right. & \frac{\beta_{1}(1-\eta) \bar{v}_{1}}{\bar{P}}\left[\frac{\beta_{2}(1-\eta)(\bar{x}+\bar{y})}{\left(a_{z}+\delta_{2} \bar{I}\right) \bar{P}}\right. \\
& \left.\left.-\frac{\left(a_{w}+\delta_{3} \bar{I}\right) d_{2}}{k_{2}(1-\varepsilon)\left(a_{z}+\delta_{2} \bar{I}\right)}\right]\right\} \bar{v}_{2}=0
\end{aligned}
$$

Eq. (25) has two possible solutions. If $\bar{v}_{2}=0$, then from Eq. 22) one has that $\bar{v}_{1}=0$ or $\bar{z}=0$. 
Note that $\bar{v}_{1}=0$ leads to the virus free equilibrium $E_{0}=\left(x^{*}, 0,0,0,0,0, I^{*}\right)$. If $\bar{z}=0$, we obtain the HBV-only persistence equilibrium $\bar{E}=$ $\left(\bar{x}, \bar{y}, 0,0, \bar{v}_{1}, 0, \bar{I}\right)$ which has biological significance if $\mathcal{R}_{0}>1$. If $\bar{v}_{2} \neq 0$, the model admits a coinfection equilibrium $\hat{E}=\left(\hat{x}, \hat{y}, \hat{z}, \hat{w}, \hat{v}_{1}, \hat{v}_{2}, \hat{I}\right)$ where the existence is verified numerically.

3) Stability of equilibria: We have the following result.

Theorem 6. If $\mathcal{R}_{0}<1$, then the infection free equilibrium $E_{0}$ of model system (19) is globally asymptotically stable in $\Sigma$ whenever

$$
\begin{gathered}
k_{3}(1-\varepsilon)\left(a_{y}+\delta_{1} I^{*}\right)+\frac{k_{1}(1-\varepsilon) k_{2}(1-\varepsilon) \beta_{2}(1-\eta)}{d_{2}} \\
\quad-k_{1}(1-\varepsilon)\left(a_{w}+\delta_{3} I^{*}\right) \geq 0 .
\end{gathered}
$$

Proof: Consider the following LaSalle function candidate:

$$
L(t)=a y+a z+a w+b v_{1}+c v_{2},
$$

where $\mathrm{a}, \mathrm{b}$ and $\mathrm{c}$ are positive constants to be determined later. Using the fact that $x \leq x^{*}$ and $I \geq I^{*}$, the derivative of $L$ along the solution of (19) satisfies

$$
\begin{aligned}
\dot{L}= & \frac{a \beta_{1}(1-\eta) x v_{1}}{P}+\frac{a \beta_{2}(1-\eta) x v_{2}}{P}-a\left(a_{y}+\delta_{1} I\right) y \\
& -a\left(a_{z}+\delta_{2} I\right) z-a\left(a_{w}+\delta_{3} I\right) w+b k_{1}(1-\varepsilon) y \\
& +b k_{3}(1-\varepsilon) w-b d_{1} v_{1}+c k_{2}(1-\varepsilon) w-c d_{2} v_{2} \\
\leq & {\left[a \beta_{1}(1-\eta)-b d_{1}\right] v_{1} } \\
& +\left[b k_{1}(1-\varepsilon)-a\left(a_{y}+\delta_{1} I^{*}\right)\right] y \\
& +\left[a \beta_{2}(1-\eta)-c d_{2}\right] v_{2} \\
& +\left[b k_{3}(1-\varepsilon)+c k_{2}(1-\varepsilon)-a\left(a_{w}+\delta_{3} I^{*}\right)\right] w .
\end{aligned}
$$

We choose $a=k_{1}(1-\varepsilon), b=a_{y}+\delta_{1} I^{*}$ and $c=\frac{k_{1}(1-\varepsilon) \beta_{2}(1-\eta)}{d_{2}}$ so that the coefficients of $y, v_{2}$ and $w$ are equal to zero. In this case, if the condition (26) holds, we obtain

$$
\dot{L} \leq\left(a_{y}+\delta_{1} I^{*}\right)\left[\mathcal{R}_{0}-1\right] v_{1} .
$$

Thus, if $\mathcal{R}_{0} \leq 1$ then $\dot{L} \leq 0$ $\forall x, y, z, w, v_{1}, v_{2}, I \geq 0$ and $\dot{L}=0$ if only if $\left(x, y, z, w, v_{1}, v_{2}\right)=\left(x^{*}, 0,0,0,0,0, I^{*}\right)$. Then the globally asymptotically attractivity of $E_{0}$ follows from Lyapunov LaSalle Invariance Principle [18]. This completes the proof.

Now, we study the stability of the HBV-only persistence equilibrium $\bar{E}=\left(\bar{x}, \bar{y}, 0,0, \bar{v}_{1}, 0, \bar{I}\right)$. The Jacobian matrix of model system $(19)$ at $\bar{E}$ is

$$
J_{\bar{E}}=\left(\begin{array}{ccccc}
-d_{x}-S_{2} & S_{1} & S_{1} \\
S_{2} & -S_{1}-a_{y}-\delta_{1} \bar{I} & -S_{1} & \\
0 & \multicolumn{2}{c}{0} & -S_{1}-a_{z}-\delta_{2} \bar{I} \\
0 & 0 & S_{1} & \\
0 & k_{1}(1-\varepsilon) & 0 & \\
0 & \multicolumn{2}{c}{0} & 0 & \\
0 & \multicolumn{2}{c}{\rho_{1} \bar{I}} & \rho_{2} \bar{I} & \\
& S_{1} & -S_{3} & -S_{4} & 0 \\
& -S_{1} & S_{3} & -S_{5} & -\delta_{1} \bar{y} \\
& 0 & 0 & S_{4} & 0 \\
-a_{w}-\delta_{3} \bar{I} & 0 & S_{5} & 0 \\
k_{3}(1-\varepsilon) & -d_{1} & 0 & 0 \\
k_{2}(1-\varepsilon) & 0 & -d_{2} & 0 \\
\rho_{3} \bar{I} & 0 & 0 & -q \bar{I}
\end{array}\right),
$$

where $S_{1}=\frac{\beta_{1}(1-\eta) \bar{x} \bar{v}_{1}}{(\bar{x}+\bar{y})^{2}}, S_{2}=\frac{\beta_{1}(1-\eta) \bar{y} \bar{v}_{1}}{(\bar{x}+\bar{y})^{2}}$, $S_{3}=\frac{\beta_{1}(1-\eta) \bar{x}}{\bar{x}+\bar{y}}, S_{4}=\frac{\beta_{2}(1-\eta) \bar{x}}{\bar{x}+\bar{y}}$ and $S_{5}=$ $\frac{\beta_{2}(1-\eta) \bar{y}}{\bar{x}+\bar{y}}$. The local stability of $\bar{E}$ is governed by the eigenvalues of the $J_{\bar{E}}$. Hence, conditions for local stability of $\bar{E}$ have been derived by applying the Routh-Hurwitz criterion to the characteristic equation of $J_{E}$. The expresions are complicated and are not presented here, but available from the authors on request. Importantly, the set of parameters satisfying these conditions is not empty.

Figure 1 presents the time evolution of model system (19) when $\beta_{1}=0.02 \beta_{2}=0.07, \varepsilon=\eta=$ $0, k_{1}=600$ and $\rho_{1}=0.00002$ (so that $\mathcal{R}_{0}>1$ ). All other parameter values are as in Table 1 . In this case, the co-infection equilibrium is $\hat{E}(6.5 \times$ $10^{4}, 1.8 \times 10^{4}, 490.93,310.3,1.572 \times 10^{7}, 1.55 \times$ $\left.10^{5}, 670.9\right)$. Initial conditions have been chosen to be $x(0)=2 \times 10^{7}, y(0)=10^{4}, z(0)=4 \times 10^{4}$, $w(0)=3 \times 10^{3}, v_{1}(0)=2 \times 10^{3}, v_{2}(0)=1.5 \times$ 
P. T. Mouofo, J. J. Tewa, S. Bowong, Modelling and analysis of a within-host model of hepatitis ...
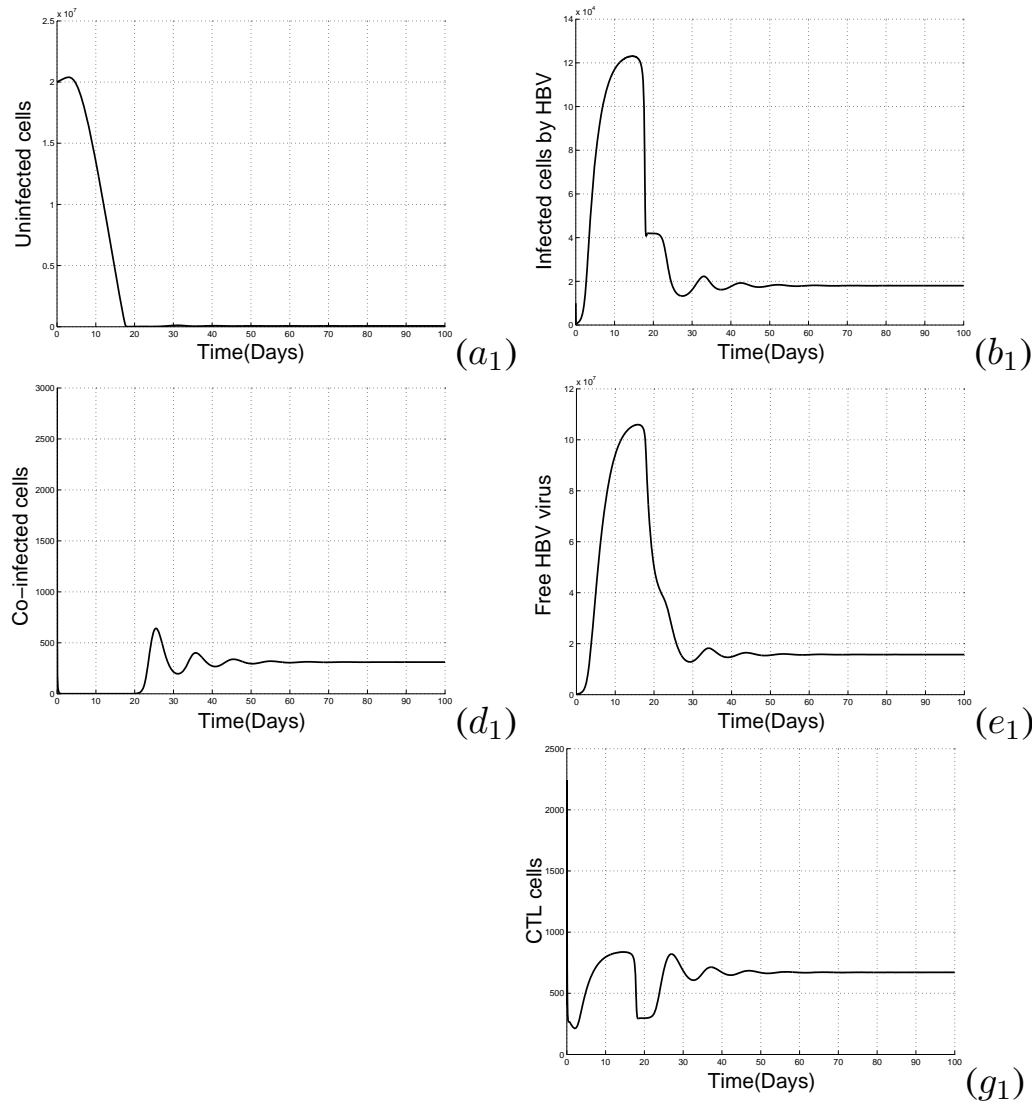
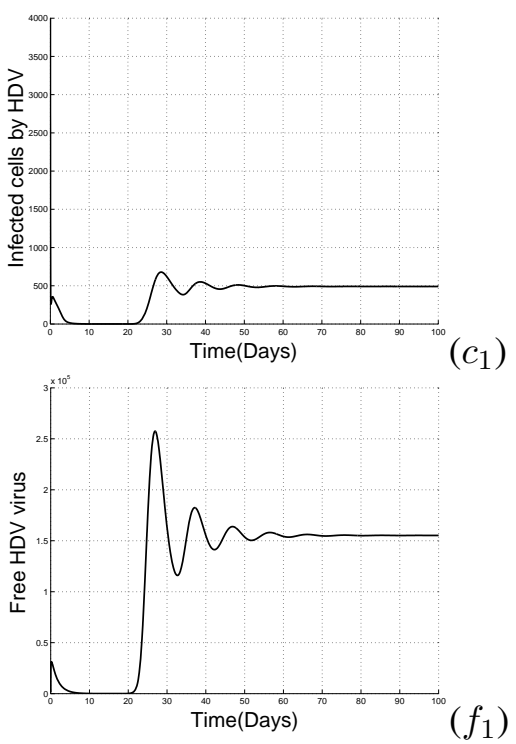

Fig. 1. Time evolution of model system (19) when $\beta_{1}=0.02 \beta_{2}=0.07, \varepsilon=\eta=0, k_{1}=600$ and $\rho_{1}=0.00002$ (so that $\mathcal{R}_{0}>1$ ). All other parameter values are as in Table 1 .

$10^{3}$ and $I(0)=4 \times 10^{2}$. From this figure, it is evident that the trajectories of model system (19) converge to the co-infection equilibrium.

\section{OPtimal CONTROL OF TREATMENT IN THE HB V-HDV CO-INFECTION MODEL}

This section deals with the problem of optimal control of the co-infection of HBV and HDV. More precisely, we are concerned with the problem of adopting the best strategy of treatment to fight the HBV-HDV co-infection. We seek to search a maximum count of healthy cells with a minimum dose of the administered drugs. Hence, if we denote $\eta(t)$ the first control variable which is efficacy of inhibiting new virus infections as a consequence of virus clearance and $\varepsilon(t)$ the second control variable which represents the efficacy of inhibiting viral production from infected cells, model system (19) can be written, to accommodate control actions or chemotherapy treatment, as follows:

$$
\left\{\begin{array}{l}
\dot{x}=\lambda-d_{x} x-\frac{\beta_{1}(1-\eta(t)) x v_{1}}{P}-\frac{\beta_{2}(1-\eta(t)) x v_{2}}{P}, \\
\dot{y}=\frac{\beta_{1}(1-\eta(t)) x v_{1}}{P}-\frac{\beta_{2}(1-\eta(t)) y v_{2}}{P}-a_{y} y \\
\dot{z}=\frac{\beta_{2}(1-\eta(t)) x v_{2}}{P}-\frac{\beta_{1}(1-\eta(t)) z v_{1}}{P}-\delta_{z} z I, \\
\dot{w}=\frac{\beta_{2}(1-\eta(t)) y v_{2}}{P}+\frac{\beta_{1}(1-\eta(t)) z v_{1}}{P}-a_{w} w \\
\dot{v_{1}}=k_{1}(1-\varepsilon(t)) y+k_{3}(1-\varepsilon(t)) w-d_{1} v_{1}, \\
\dot{v}_{2}=k_{2}(1-\varepsilon(t)) w-d_{2} v_{2}, \\
\dot{I}=\rho_{1} y I+\rho_{2} z I+\rho_{3} w I+p I-q I^{2},
\end{array}\right.
$$


P. T. Mouofo, J. J. Tewa, S. Bowong, Modelling and analysis of a within-host model of hepatitis ...

with $x^{0}, y^{0}, z^{0}, w^{0}, v_{1}^{0}, v^{0}$ and $I^{0}$ the given initial values of $x, y, w, z, v_{1}, v_{2}, I$ at $t_{0}=0$ respectively. The control functions, $\eta(t)$ and $\varepsilon(t)$, are bounded, Lebesgue integrable functions.

Our objective functional to be maximized is

$$
J(\eta, \varepsilon)=\int_{0}^{t_{f}}\left[x(t)-\left(\frac{A}{2} \eta^{2}+\frac{B}{2} \varepsilon^{2}\right)\right] d t .
$$

In other words, we want to maximize the benefit based on the healthy liver cells count and minimizing the cost based on the percentage effect chemotherapy given (i.e. $\eta$ and $\varepsilon$ ). The parameters $A$ and $B$ are positive and represent the weights on the benefit and cost.

The goal is to seek an optimal control pair $\left(\eta^{*}, \varepsilon^{*}\right)$ such that

$$
J\left(\eta^{*}, \varepsilon^{*}\right)=\max \{J(\eta, \varepsilon):(\eta, \varepsilon) \in \mathcal{U}\},
$$

where $\mathcal{U}$ is the control set defined by:

$$
\begin{gathered}
\mathcal{U}=\{u=(\eta, \varepsilon), \eta, \varepsilon \text { measurable }, 0 \leq \eta(t) \leq \\
\left.1,0 \leq \varepsilon(t) \leq 1, \forall t \in\left[0, t_{f}\right]\right\} .
\end{gathered}
$$

The basic framework of this problem is to prove the existence and the uniqueness of the optimal control and to characterize it.

\section{A. Analysis of Optimal Controls}

The necessary conditions that an optimal pair must satisfy come from Pontryagin's Maximum Principle [21]. This principle converts (28) - 30. into a problem of minimizing pointwise a Hamiltonian, $H$, with respect to $\eta$ and $\varepsilon$ :

$$
\begin{aligned}
& H=x(t)-\left(\frac{A}{2} \eta^{2}+\frac{B}{2} \varepsilon^{2}\right) \\
& +\lambda_{1}\left[\lambda-d_{x} x-\frac{\beta_{1}(1-\eta(t)) x v_{1}}{P}-\frac{\beta_{2}(1-\eta(t)) x v_{2}}{P}\right] \\
& +\lambda_{2}\left[\frac{\beta_{1}(1-\eta(t)) x v_{1}}{P}-\frac{\beta_{2}(1-\eta(t)) y v_{2}}{P}-a_{y} y\right. \\
& \left.-\delta_{1} y I\right]+\lambda_{3}\left[\frac{\beta_{2}(1-\eta(t)) x v_{2}}{P}-\frac{\beta_{1}(1-\eta(t)) z v_{1}}{P}\right. \\
& \left.-a_{z} z-\delta_{2} z I\right]+\lambda_{4}\left[\frac{\beta_{2}(1-\eta(t)) y v_{2}}{P}\right.
\end{aligned}
$$

$$
\begin{aligned}
& \left.+\frac{\beta_{1}(1-\eta(t)) z v_{1}}{P}-a_{w} w-\delta_{3} w I\right] \\
& +\lambda_{5}\left[k_{1}(1-\varepsilon(t)) y+k_{3}(1-\varepsilon(t)) w-d_{1} v_{1}\right] \\
& +\lambda_{6}\left[k_{2}(1-\varepsilon(t)) w-d_{2} v_{2}\right] \\
& +\lambda_{7}\left[\rho_{1} y I+\rho_{2} z I+\rho_{3} w I+p I-q I^{2}\right] .
\end{aligned}
$$

By applying Pontryagins Maximum Principle [21] and the existence result for the optimal control pairs from [22], we have the following result.

Theorem 7. There exists an optimal control pair $\eta^{*}, \varepsilon^{*}$ and corresponding solution $x^{*}, y^{*}, z^{*}, w^{*}$, $v_{1}^{*}, v_{2}^{*}$ and $I^{*}$, such that $J\left(\eta^{*}, \varepsilon^{*}\right)=\max _{\mathcal{U}} J(\eta, \varepsilon)$. Furthermore, there exist adjoint functions $\lambda_{1}, \lambda_{2}$, $\lambda_{3}, \lambda_{4}, \lambda_{5}, \lambda_{6}$, and $\lambda_{7}$ such that equation (32) holds with the transversality conditions

$$
\lambda_{i}\left(t_{f}\right)=0, \text { for } i=1, \ldots, 7
$$

and $P=x^{*}+y^{*}+z^{*}+w^{*}$. The following characterization holds:

$$
\begin{aligned}
& \eta^{*}=\min \left\{\max \left\{0, R_{1}(t)\right\}, 1\right\}, \\
& \varepsilon^{*}=\min \left\{\max \left\{0, R_{2}(t)\right\}, 1\right\}
\end{aligned}
$$

where

$$
\begin{array}{r}
R_{1}(t)=\frac{1}{A}\left[\lambda_{1} \frac{\beta_{1} x^{*} v_{1}^{*}+\beta_{2} x^{*} v_{2}^{*}}{P}\right. \\
+\lambda_{2} \frac{\beta_{2} y^{*} v_{2}^{*}-\beta_{1} x^{*} v_{1}^{*}}{P}+\lambda_{3} \frac{\beta_{1} z^{*} v_{1}^{*}-\beta_{2} x^{*} v_{2}^{*}}{P} \\
\left.-\lambda_{4} \frac{\beta_{1} z^{*} v_{1}^{*}+\beta_{2} y^{*} v_{2}^{*}}{P}\right] \\
R_{2}(t)=\frac{-\lambda_{5}\left(k_{1} y^{*}+k_{3} w^{*}\right)-\lambda_{6} k_{2} w^{*}}{B}
\end{array}
$$

Proof: Corollary 4.1 in Fleming and Rishel [22] gives the existence of an optimal control pair due to the concavity of the integrand of $J$ with respect to $(\eta, \varepsilon)$, a priori boundedness of the state solutions, and the Lipschitz property of the state system with respect to the state variables. Applying Pontryagin's Maximum Principle, we obtain 
P. T. Mouofo, J. J. Tewa, S. Bowong, Modelling and analysis of a within-host model of hepatitis ...

$$
\begin{aligned}
& \dot{\lambda}_{1}=-1+\lambda_{1}\left(d_{x}+\frac{\beta_{1}(1-\eta) v_{1}^{*}\left(y^{*}+z^{*}+w^{*}\right)}{P^{2}}+\frac{\beta_{2}(1-\eta) v_{2}^{*}\left(y^{*}+z^{*}+w^{*}\right)}{P^{2}}\right), \\
& -\lambda_{2}\left(\frac{\beta_{1}(1-\eta) v_{1}^{*}\left(y^{*}+z^{*}+w^{*}\right)}{P^{2}}+\frac{\beta_{2}(1-\eta) y^{*} v_{2}^{*}}{P^{2}}\right) \\
& -\lambda_{3}\left(\frac{\beta_{2}(1-\eta) v_{2}^{*}\left(y^{*}+z^{*}+w^{*}\right)}{P^{2}}+\frac{\beta_{1}(1-\eta) z^{*} v_{1}^{*}}{P^{2}}\right) \\
& -\lambda_{4}\left(\frac{\beta_{2}(1-\eta) y^{*} v_{2}^{*}}{P^{2}}+\frac{\beta_{1}(1-\eta) z^{*} v_{1}^{*}}{P^{2}}\right) \\
& \dot{\lambda}_{2}=-\lambda_{1}\left(\frac{\beta_{1}(1-\eta) x^{*} v_{1}^{*}}{P^{2}}+\frac{\beta_{2}(1-\eta) x^{*} v_{2}^{*}}{P^{2}}\right)+\lambda_{3}\left(\frac{\beta_{2}(1-\eta) x^{*} v_{2}^{*}}{P^{2}}-\frac{\beta_{1}(1-\eta) z^{*} v_{1}^{*}}{P^{2}}\right) \\
& +\lambda_{2}\left(\frac{\beta_{1}(1-\eta) x^{*} v_{1}^{*}}{P^{2}}+\frac{\beta_{2}(1-\eta) v_{2}^{*}\left(x^{*}+z^{*}+w^{*}\right)}{P^{2}}+a_{y}+\delta_{1} I^{*}\right) \\
& +\lambda_{4}\left(\frac{\beta_{1}(1-\eta) z^{*} v_{1}^{*}}{P^{2}}-\frac{\beta_{2}(1-\eta) v_{2}^{*}\left(x^{*}+z^{*}+w^{*}\right)}{P^{2}}\right)-k_{1}(1-\varepsilon) \lambda_{5}-\rho_{1} I^{*} \lambda_{7}, \\
& \dot{\lambda}_{3}=-\lambda_{1}\left(\frac{\beta_{1}(1-\eta) x^{*} v_{1}^{*}}{P^{2}}+\frac{\beta_{2}(1-\eta) x^{*} v_{2}^{*}}{P^{2}}\right)+\lambda_{2}\left(\frac{\beta_{1}(1-\eta) x^{*} v_{1}^{*}}{P^{2}}-\frac{\beta_{2}(1-\eta) y^{*} v_{2}^{*}}{P^{2}}\right) \\
& +\lambda_{3}\left(\frac{\beta_{2}(1-\eta) x^{*} v_{2}^{*}}{P^{2}}+\frac{\beta_{1}(1-\eta) v_{1}^{*}\left(x^{*}+y^{*}+w^{*}\right)}{P^{2}}+a_{z}+\delta_{2} I^{*}\right) \\
& +\lambda_{4}\left(\frac{\beta_{2}(1-\eta) y^{*} v_{2}^{*}}{P^{2}}-\frac{\beta_{1}(1-\eta) v_{1}^{*}\left(x^{*}+y^{*}+w^{*}\right)}{P^{2}}\right)-\rho_{2} I^{*} \lambda_{7}, \\
& \dot{\lambda}_{4}=-\lambda_{1}\left(\frac{\beta_{1}(1-\eta) x^{*} v_{1}^{*}}{P^{2}}+\frac{\beta_{2}(1-\eta) x^{*} v_{2}^{*}}{P^{2}}\right)+\lambda_{2}\left(\frac{\beta_{1}(1-\eta) x^{*} v_{1}^{*}}{P^{2}}-\frac{\beta_{2}(1-\eta) y^{*} v_{2}^{*}}{P^{2}}\right) \\
& +\lambda_{3}\left(\frac{\beta_{2}(1-\eta) x^{*} v_{2}^{*}}{P^{2}}-\frac{\beta_{1}(1-\eta) z^{*} v_{1}^{*}}{P^{2}}\right)-k_{3}(1-\varepsilon) \lambda_{5}-k_{2}(1-\varepsilon) \lambda_{6}-\rho_{3} I^{*} \lambda_{7} \\
& +\lambda_{4}\left(\frac{\beta_{2}(1-\eta) y^{*} v_{2}^{*}}{P^{2}}+\frac{\beta_{1}(1-\eta) z^{*} v_{1}^{*}}{P^{2}}+a_{w}+\delta_{3} I^{*}\right), \\
& \dot{\lambda}_{5}=\frac{\beta_{1}(1-\eta) x^{*}}{P}\left(\lambda_{1}-\lambda_{2}\right)+\frac{\beta_{1}(1-\eta) z^{*}}{P}\left(\lambda_{3}-\lambda_{4}\right)+d_{1} \lambda_{5}, \\
& \dot{\lambda}_{6}=\frac{\beta_{2}(1-\eta) x^{*}}{P}\left(\lambda_{1}-\lambda_{3}\right)+\frac{\beta_{2}(1-\eta) y^{*}}{P}\left(\lambda_{2}-\lambda_{4}\right)+d_{2} \lambda_{6}, \\
& \dot{\lambda}_{7}=\delta_{1} y^{*} \lambda_{2}+\delta_{2} z^{*} \lambda_{3}+\delta_{3} w^{*} \lambda_{4}-\left(\rho_{1} y^{*}+\rho_{2} z^{*}+\rho_{3} w^{*}+p-2 q I^{*}\right) \lambda_{7},
\end{aligned}
$$


P. T. Mouofo, J. J. Tewa, S. Bowong, Modelling and analysis of a within-host model of hepatitis ...

$$
\dot{\lambda}_{1}=-\frac{\partial H}{\partial x}, \dot{\lambda}_{2}=-\frac{\partial H}{\partial y}, \ldots, \dot{\lambda}_{7}=-\frac{\partial H}{\partial I},
$$

evaluated at the optimal control pair and corresponding states, which results in the stated adjoint system (32) and (33) [35]. By considering the optimality conditions,

$$
\frac{\partial H}{\partial \eta}=0 \text { and } \frac{\partial H}{\partial \varepsilon}=0
$$

and solving for $\eta^{*}, \varepsilon^{*}$, subject to the constraints, the characterizations (34) can be derived. To illustrate the characterization of $\varepsilon^{*}$, we have

$$
\frac{\partial H}{\partial \varepsilon}=-B \varepsilon-\lambda_{5}\left(k_{1} y+k_{3} w\right)-\lambda_{6} k_{2} w=0
$$

at $\varepsilon^{*}$ on the set $\left\{t \mid 0<\varepsilon^{*}(t)<1\right\}$. On this set, we have

$\varepsilon^{*}(t)=\frac{-\lambda_{5}\left(k_{1} y+k_{3} w\right)-\lambda_{6} k_{2} w}{B}$. Taking into account the bounds on $\varepsilon$, we obtain the characterization of $\varepsilon$ in (34).

Next, we discuss the numerical solutions of the optimality system and the corresponding optimal control pairs, the parameter choices, and the interpretations from various cases.

\section{B. Numerical results}

In this section, we study numerically an optimal treatment strategy of our HBV-HDV co-infection model. The optimal treatment strategy is obtained by solving the optimality system, consisting of 14 ODEs from the state and adjoint equations. An iterative method is used for solving the optimality system. We start to solve the state equations with a guess for the controls over the simulated time using a forward fourth order Runge-Kutta scheme. Because of the transversality conditions (33), the adjoint equations are solved by a backward fourth order Runge-Kutta scheme using the current iteration solution of the state equations. Then, the controls are updated by using a convex combination of the previous controls and the value from the characterizations (34). This process is repeated and iteration is stopped if the values of unknowns at the previous iteration are very close to the ones at the present iteration.

In order to evaluate our control strategy, we consider six co-infected patients by $\mathrm{HBV}$ and
HDV: $A_{1}, A_{2}, B_{1}, B_{2}, C_{1}$, and $C_{2}$ divided in three groups such that The first group consists of $A_{1}$ and $A_{2}$ and the viral HDV load of patients $A_{1}$ and $A_{2}$ are the same and are $1.16 \times 10^{3}$ copies $/ \mathrm{ml}$. The second group are patients $B_{1}$ and $B_{2}$ with the same HDV viral loads of $1.16 \times 10^{5}$ copies $/ \mathrm{ml}$; the end group are patients $C_{1}$, and $C_{2}$ with high levels of HDV viremia which are $1.16 \times 10^{7}$ copies/ml. We suppose that patients $A_{1}, B_{1}$ and $C_{1}$ are not under treatment. Otherwise, patients $A_{2}, B_{2}$ and $C_{2}$ are under our treatment control strategy. We want to evaluate in 100 days the evolution of viral HDV load in every patient. We suppose that the viral load of HBV in those groups are $10^{8}$ copies $/ \mathrm{ml}$. Note that some studies report improvements in patients, with interferon- $\alpha$ (IFN) efficacy as high as $90 \%(\eta=0.9)$ [36]. Although lamivudine (LMV) does not have a direct effect on HDV viral production $k_{2}$, its effect on the viral production of HBV will also have an effect on the HDV viral dynamics. Studies such as Lewin et al [37] show efficacy levels of therapies based on LMV varying between $90 \%$ to $99 \%(\varepsilon>0.9)$ for the HBV infection. With this in mind, we consider combination of interferon- $\alpha$ and lamivudine in our simulations.

In Fig.(2), parameter values are the same as in Fig.(1). Figure (2) $\left(a_{1}\right)-\left(c_{1}\right)$ presents the time evolution of viral load of patients of group 1. In this figure, we observe that when there is not strategy of treatment, the disease persists in the host. But, with our strategy of treatment, the disease disappear in the host. To have this result, the control $\varepsilon(t)$ is at it maximal value almost all the period of our control strategy. This means that, to control HDV, we need medication with hight efficacy. We have the same observation on the other figures for group 2 and group 3.

Fig.(3) illustrate the importance of immune system in our model. We observe that without CTL cells, the viral load of patients $A_{1}, B_{1}$ and $C_{1}$ increase. Otherwise, the viral load of patients $A_{2}, B_{2}$ and $C_{2}$ converge to zero if only if $\varepsilon(t)$ and $\eta(t)$ are to their maximal values during all the period of control strategy. So, it is difficult 
P. T. Mouofo, J. J. Tewa, S. Bowong, Modelling and analysis of a within-host model of hepatitis ...
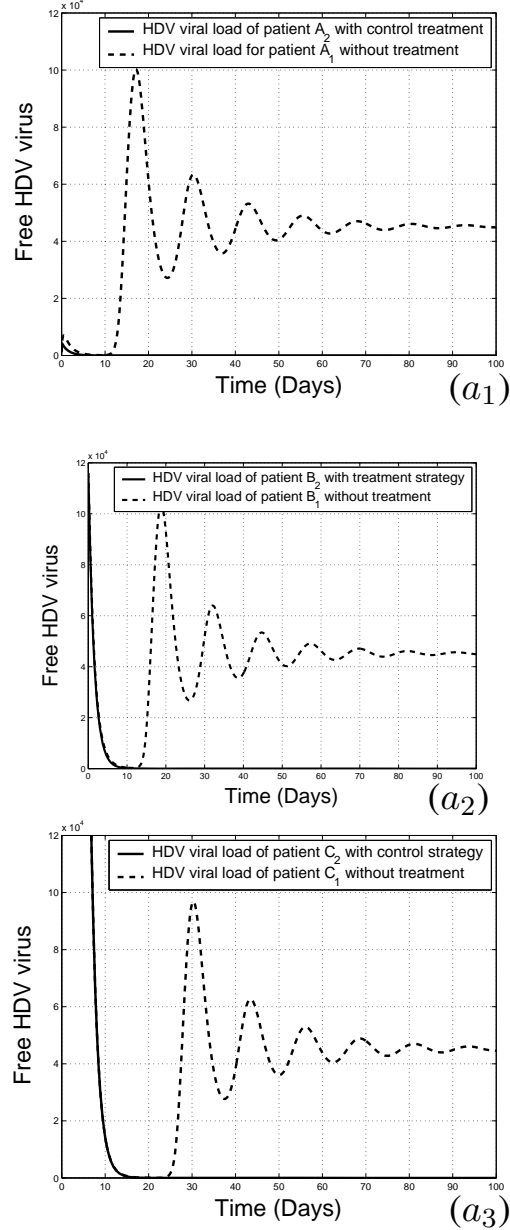
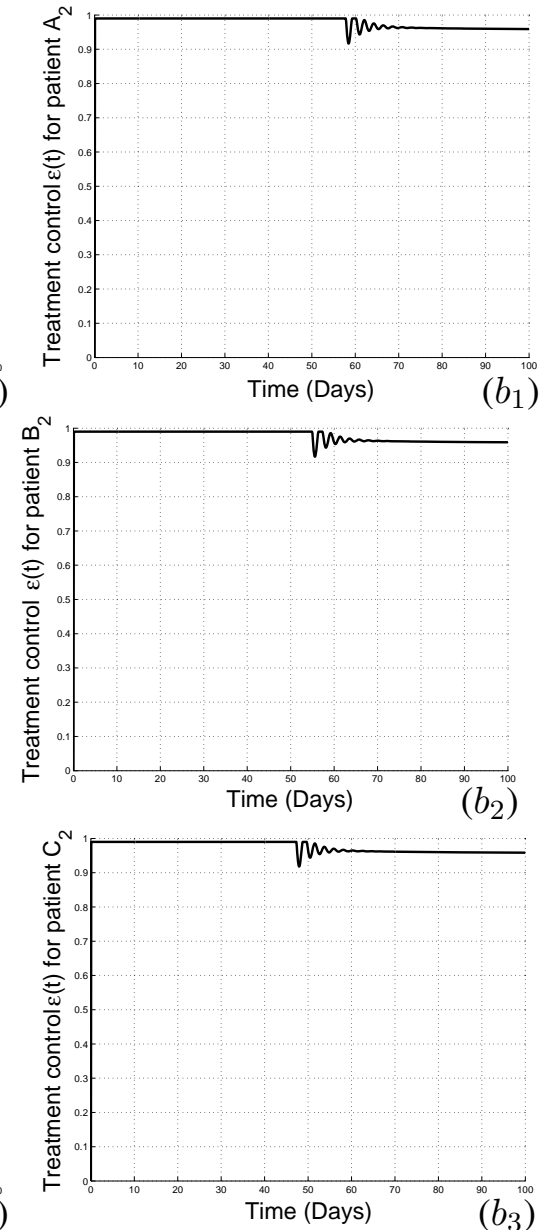
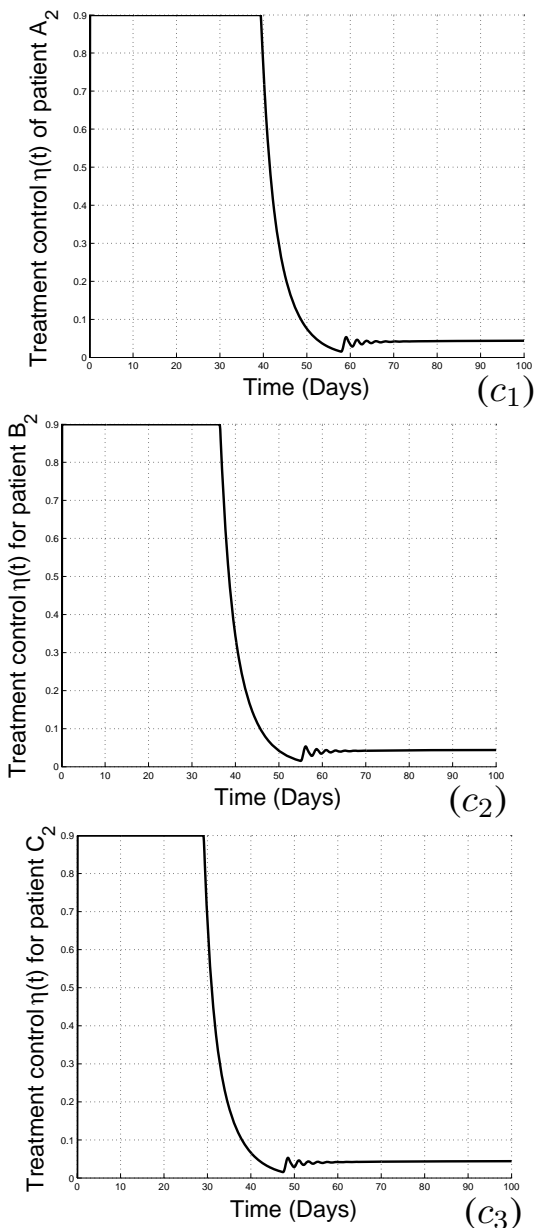

Fig. 2. Time evolution of viral load of patients $A_{1}, B_{1}, C_{1}$ without treatment, and patients $A_{2}, B_{2}$ and $c_{2}$ with our treatment strategy.

to control the disease in this case. This show the importance of immune system in our model.

\section{DISCUSSION AND CONCLUSION}

Hepatitis D virus infection has a worldwide distribution. Studying the transmission, epidemiology and dynamic virus of HDV infection is an important topic. It is a unique virus for which many open questions remain. For example, HDVspecific treatment protocols still do not exist. The investigation of the inter-related dynamics of chronic HBV and HDV infections are important to understanding how treatment may affect this complex system. To this end, the development of biologically realistic mathematical models is an important tool. The main objective of this paper was to shed light on the interaction between HBV and HDV.

A realistic deterministic ODE based compartmental model for the transmission of $\mathrm{HBV}$ and HDV co-dynamics within the body of a host has been proposed and analyzed. The HBV-only model was qualitatively examined, first of all.

The mathematical analysis results show that the basic reproductive number $\mathcal{R}_{0}$ of the cointeraction HBV-HDV model is the same than the basic reproduction number of the HBV model alone. This suggests that the eradication of HDV is conditioned by the eradication of HBV. The epidemiological implication of this is that for 
P. T. Mouofo, J. J. Tewa, S. Bowong, Modelling and analysis of a within-host model of hepatitis ...
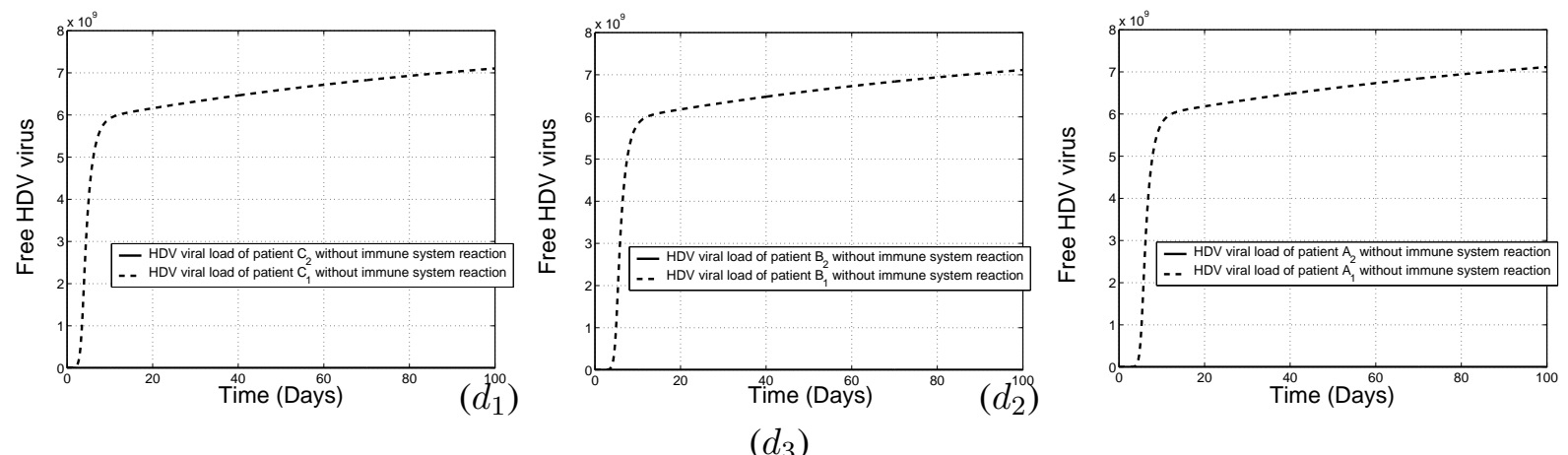

Fig. 3. Time evolution of viral load different groups without CTL cells .

the effective eradication and control of HDV, $\mathcal{R}_{0}$ should be less than one. Moreover, achieving this may be too costly, because it means that for constant controls, one needs to keep treating for infinite time. Therefore, we considered time dependent controls as a way out, to ensure the eradication of the disease in a finite time. In this light, we addressed the optimal control by deriving and analyzing the conditions for optimal eradication of the disease. From our numerical results, we can conclude that immune responses play a significant role in eradication of disease. Moreover, to eradicate the disease, it is important to manufacture a drug treatment with hight efficacy which can block the viral production.

\section{REFERENCES}

[1] Hoofnagle J, Mullen K, Peters M (1987);“ Treatment of chronic delta hepatitis with recombinant alpha interferon". In: Rizzetto M, Gerin JL, Purcell RH, editors. The hepatitis delta virus and its infection. New York: Alan R. Liss.

[2] Lau DT, Kleiner DE, Park Y, Bisceglie AMD, Hoofnagle JH (1999); "Resolution of chronic delta hepatitis after 12 years of interferon alfa therapy". Gastroenterology 117(5): 12291233.

[3] Lau DT, Doo E, Park Y, Kleiner DE, Schmid P, et al. (1999); "Lamivudine for chronic delta hepatitis. Hepatology”. 30(2): 546549.

[4] Yurdaydin C, Bozkaya H, Gurel S, Tillman HL, Aslan N, et al. (2002); "Famciclovir treatment of chronic delta hepatitis". Journal Hepatology 37(2): 266271

[5] R. Qesmi, J. Wu, J. M. Heffernan, (2010); “Influence of backward bifurcation in a model of hepatitis $B$ and $C$ viruses". Math. Biosci. 224, 118125.

[6] K. Wang, W. Wang,(2007); "Propagation of HBV with spatial dependence". Math. Biosci. 210, 7895
[7] F. Brauer, C. Castillo-Chvez,(2001); "Mathematical Models in Population Biology and Epidemiology, Texts in Applied Mathematics". Springer, New York, NY, .

[8] Murray J. M., Purcell R. H., Wieland S. F. (2006); "The half-life of hepatitis B virions". Hepatology 44, 11171121.

[9] M. A. Nowak, R. M. May,, (2000); "Virus Dynamics". Oxford University Press, Oxford.

[10] L. Min, Y. Su, Y. Kuang, (2008); "Mathematical Analysis of a Basic Virus Infection Model With Application to HBV Infection". Rocky Mountain Journal of Mathematics, 38, 113.

[11] K. Wang, W. Wang, S. Song,(2008); "Dynamics of an HBV model with diffusion and delay", J. Theor. Bio. 253 3644.

[12] S. Eikenberry, S. Hews, J. Nagy, Y. Kuang, (2009); "The dynamics of a delay model of hepatitis B virus infection with logistic hepatocyte growth", Math. Biosci. Eng. 6 283299, .

[13] Xiridou M, Borkent-Raven B, Hulshof J, Wallinga J (2009);"How hepatitis D virus can hinder the control of hepatitis B virus". PLoS ONE 4(4): e5247. doi:10.1371/journal.pone.0005247.

[14] Whalley S. A., Murray J. M., Brown D., Webster G. J. M., Emery V. C., Dusheiko G. M., Perelson A. S. (2001); "Kinetics of Acute Hepatitis B Virus Infection in Humans", J. Exp. Med. 193, 847-853.

[15] Bralet M., Branchereau S., Brechot C. and Ferry N. (1994);" Cell Lineage Study in the Liver Using Retroviral Mediated Gene Transfer"; Am. J. Pathol. 144, 896-905.

[16] S. A. Gourley, Y. Kuang, and J. D. Nagy, "Dynamics of a delay differential equation model of hepatitis $B$ virus infection", Journal of Biological Dynamics, vol. 2, no. 2, pp. 140153, 2008.

[17] P. van den Driessche, J. Watmough,(2002); "Reproduction numbers and sub-threshold endemic equilibria for compartmental models of disease transmission", Math. Biosci. 180 29-48. 
[18] Bhatia NP, Szeg GP.1970; "Stability theory of dynamical systems". Springer-Verlag.

[19] M. Schaper, F. Rodriguez-Frias, R. Jardi, D. Tabernero, M. Homs, G. Ruiz, J. Quer, R. Esteban, M. Buti,(2010); Quantitative longitudinal evaluations of hepatitis delta virus rna and hepatitis B virus DNA shows a dynamic, complex replicative profile in chronic hepatitis $B$ and $D$, Journal of Hepatology 52658.

[20] R. Jardi, F. Rodriguez, M. Buti, X. Costa, M. Cotrina, R. Galimany, R. Esteban, J. Guardia, (2001); Role of hepatitis $B, C$, and $D$ viruses in dual and triple infection: Influence of viral genotypes and hepatitis $B$ precore and basal core promoter mutations on viral replicative interference, Hepatology 34404.

[21] L. S. Pontryagin, V. G. Boltyanskii, R. V. Gamkrelidze, and E. F. Mishchenko, (1962) "The mathematical theory of optimal processes", Wiley, New York.

[22] W. H. Fleming and R. W. Rishel, (1975); "Deterministic and Stochastic Optimal Control", Springer Verlag, New York.

[23] J. A. Deans, S. Cohen, (1983); "Immunology of malaria. Ann. Rev. Microbiol., 37 , 25-50.

[24] A. M. Elaiw,(2015) "Global stability analysis of humoral immunity virus dynamics model including latently infected cells", J. Biol. Dyn., 9 , 215-228.

[25] A. M. Elaiw,(2015); "Global threshold dynamics in humoral immunity viral infection models including an eclipse stage of infected cells", J. Korean Soc. Ind. Appl. Math., 19 , 137-170.

[26] A. M. Elaiw, N. H. AlShamrani,(2015); "Global stability of humoral immunity virus dynamics models with nonlinear infection rate and removal", Nonlinear Anal. Real World Appl., 26 , 161-190.

[27] A. Murase, T. Sasaki, T. Kajiwara,(2005); "Stability analysis of pathogen-immune interaction dynamics", J. Math. Biol., 51, 247-267.

[28] M. A. Obaid, A. M. Elaiw,(2014); "Stability of virus infection models with antibodies and chronically infected infection models with antibodies and chronically infected cells", Abstr. Appl. Anal., 12 pages.

[29] K. HATTAF, N. YOUSFI AND A. TRIDANE; (2012)““ Global stability analysis of a generalized virus dynamics model with the immune response", Canadian Applied Mathematics Quarterly 20 (4) 499518.

[30] T. Wang, Z. Hu, F. Liao,(2014) "Stability and Hopf bifurcation for a virus infection model with delayed humoral immunity response", J. Math. Anal. Appl., 411 63-74.

[31] T. Wang, Z. Hu, F. Liao, W. Ma, "Global stability analysis for delayed virus infection model with general incidence rate and humoral immunity", Math. Comput. Simulation, 89 (2013), 13-22.

[32] S. Wang, D. Zou,(2012); "Global stability of in-host viral models with humoral immunity and intracellular delays", J. Appl. Math. Model, 36 , 1313-1322.

[33] Mostafa Khabouze, Khalid Hattaf, and Noura Yousfi1;(2014) "Stability Analysis of an Improved HBV Model with CTL Immune Response, ",International Scholarly Research Notices.

[34] Hattaf, K.; Yousfi, N. (2015); "A generalized HBV model with diffusion and two delays". Comput. Math. Appl., 69, 3140.

[35] M. I. Kamien and N. L. Schwarz,(1991); "Dynamic optimization: the calculus of variations and optimal control", North Holland, Amsterdam.

[36] Chien RN, (2008); "Current therapy for hepatitis $C$ or $D$ or immunodeficiency virus concurrent infection with chronic hepatitis B. Hepatol Int 2: 296303.

[37] Lewin SR, Ribeiro RM, Walters T, Lau GK (2001) "Analysis of hepatitis B viral load decline under potent therapy: complex decay profiles observed". Hepatology 34: 10121020.

[38] Noura Yousfi Khalid Hattaf Abdessamad Tridane, (2011); Modeling the adaptive immune response in $H B V$ infection, J. Mathematical Biology 63933957. 